CARDIOVASCULAR MEDICINE

\title{
$C$ reactive protein concentration and recurrence of atrial fibrillation after electrical cardioversion
}

\author{
O Wazni, D O Martin, N F Marrouche, M Shaaraoui, M K Chung, S Almahameed, \\ R A Schweikert, W I Saliba, A Natale
}

See end of article for authors' affiliations

Correspondence to: Dr Andrea Natale, Center for Atrial Fibrillation, Department of

Cardiovascular Medicine Cleveland Clinic

Foundation, Desk F 15

9500 Euclid Avenue,

Cleveland, $\mathrm{OH} 44195$

USA; natalea@ccf.org

Accepted 13 January 2005

Published Online First

12 May 2005
Background: To test the hypothesis that a high $\mathrm{C}$ reactive protein (CRP) concentration would predict recurrence of atrial fibrillation (AF) after cardioversion in patients taking antiarrhythmic drugs.

Methods: 111 patients who underwent direct current cardioversion for symptomatic AF were enrolled. Blood was drawn for CRP determination before cardioversion on the same day. All patients were taking antiarrhythmic drugs before and after electrical cardioversion.

Results: After a mean follow up of 76 days, 75 patients had recurrence of AF. In univariate analysis, the median CRP concentration was significantly higher in patients with AF recurrence $(3.95 \mathrm{mg} / \mathrm{l} \mathrm{v} 1.81 \mathrm{mg} / \mathrm{l}$, $p=0.002)$. Among the 55 patients with CRP in the upper 50th centile, $44(80 \%)$ experienced recurrence of AF over a total follow up of 8.98 patient years, whereas among the 56 patients with CRP in the lower 50th centile, $31(55 \%)$ experienced recurrence of AF over a total follow up of 14.3 patient years $(p<0.001)$. The adjusted hazard ratio comparing the upper 50th centile of CRP with the lower 50th centile of CRP was 2.0 (95\% confidence interval 1.2 to $3.2, p=0.007$ ).

Conclusions: CRP is independently associated with recurrence of AF after electrical cardioversion among patients taking antiarrhythmic drugs. These results suggest that inflammation may have a role in the pathogenesis of AF resistant to antiarrhythmic drugs.
A lthough ventricular rate control may be a viable treatment strategy for some patients with atrial fibrillation (AF), ${ }^{1}$ a strategy that achieves sinus rhythm may be preferable for patients with ventricular rates that are difficult to control or who remain symptomatic despite adequate ventricular rate control. ${ }^{2}$ Electrical cardioversion is an effective means to restore sinus rhythm, and the success of this procedure can be enhanced by the use of antiarrhythmic drugs. ${ }^{3}$ However, among patients taking antiarrhythmic drugs AF commonly recurs after cardioversion, usually within three months of cardioversion. ${ }^{45}$

Electrical or structural remodelling of the atria during AF may contribute to the high recurrence rates observed after cardioversion. Inflammation, necrosis, and fibrosis have been observed in atrial biopsies from patients with lone $\mathrm{AF}$ resistant to medical treatment. ${ }^{6} \mathrm{C}$ reactive protein (CRP) has been found to be increased in patients with AF and may reflect an inflammatory state that promotes $\mathrm{AF}^{7}$ A low level inflammatory response may be a part of the structural remodelling process that is associated with increased persistence of AF.

We hypothesised that increased CRP concentrations would predict recurrence of AF among patients with persistent $\mathrm{AF}$ taking antiarrhythmic drugs who undergo electrical cardioversion.

\section{METHODS}

Patients with persistent, symptomatic AF taking VaughanWilliams class I or III antiarrhythmic drugs who were followed up as outpatients at our institution and underwent successful electrical cardioversion between November 2001 and August 2002 were enrolled in the study. Blood samples for CRP, routine chemical analysis, and international normalised ratio were drawn from all patients just before the cardioversion. All patients had precardioversion ECG and transthoracic echocardiography. Exclusion criteria were surgery within 60 days, an acute coronary syndrome within
60 days, history of recent infection, and history of collagen vascular disease. Our institutional review board approved the investigation.

Patients were followed up according to the usual standard of care. In addition, after cardioversion patients were instructed to call our arrhythmia clinic if they had any palpitations. These patients were then seen in the outpatient clinic as soon as possible. Recurrence of AF was defined as AF documented by ECG at any time after the cardioversion during the study time frame. CRP concentrations were assayed by immunonephelometry by the BNII analyser protocol (Dade Behring, Deerfield, Illinois, USA). CRP concentrations were determined with a typical detection limit of $0.175 \mathrm{mg} / \mathrm{l}$ (ultrasensitive CRP).

For statistical analysis, the independent samples $t$ test was used when comparing approximately normally distributed variables between two groups. For non-normally distributed variables, the non-parametric Mann-Whitney $U$ test was used when comparing variables between two groups. Categorical variables were compared by the $\chi^{2}$ test. The relation between recurrence of $\mathrm{AF}$ and baseline predictors was assessed by Cox proportional hazards regression modelling. For regression modelling, CRP was dichotomised at the median value and hazard ratios were computed for the upper versus lower 50th centiles of CRP. Regression modelling was done by a forward selection strategy in which variables were entered into the model if $\mathrm{p}<0.20$ and retained in the model if $\mathrm{p}<0.30 .^{8}$ All data were analysed with SPSS version 9.0 (SPSS Inc, Chicago, Illinois, USA). Results with $\mathrm{p}<0.05$ were considered significant. Because the distribution of CRP was skewed to the right, CRP concentration is presented as median with interquartile range, whereas other variables are presented as mean (SD).

Abbreviations: AF, atrial fibrillation; $\mathrm{Cl}$, confidence interval; $\mathrm{CRP}$, $C$ reactive protein 


\section{RESULTS}

One hundred and eleven patients (30 women and 81 men) with persistent AF who were taking antiarrhythmic drugs were enrolled in the study. After a mean follow up of 76 days (range 0.003-298 days), 75 (68\%) patients had recurrence of AF. Table 1 presents the demographic and clinical characteristics of study participants according to AF recurrence. Both groups were comparable with respect to history of coronary artery disease and antiarrhythmic drugs used. Patients who experienced a recurrence of AF had a longer mean duration of AF but this was not significant (222 (479) days $v 203$ (345) days, $p=0.9)$ A higher proportion of patients with recurrence than with non-recurrence were women. Patients with AF recurrence tended to have larger left atria. The median CRP was significantly higher among patients with than among patients without AF recurrence (3.95 (2.19$6.13) \mathrm{mg} / \mathrm{l} v 1.81(0.78-4.17) \mathrm{mg} / \mathrm{l}, \mathrm{p}=0.002)$.

Table 2 presents characteristics of study patients and AF recurrence rates according to CRP concentration dichotomised into upper and lower 50th centiles. Patients with coronary artery disease were more likely to have increased CRP. The median CRP among those with coronary artery disease was $4.24 \mathrm{mg} / \mathrm{l}$, significantly higher than the median CRP of $2.53 \mathrm{mg} / \mathrm{l}$ among those without coronary artery disease $(p=0.007)$. Patients with CRP in the upper 50th centile had longer duration of AF (276 (546) $v 157$ (294) days, $\mathrm{p}=0.3$ ). Among the 55 patients with CRP in the upper 50th centile, $44(80 \%)$ experienced recurrence of AF over a total follow up of 8.98 patient years (AF recurrence rate of 4.9/patient year), whereas among the 56 patients with CRP in the lower 50th centile, 31 (55\%) experienced recurrence of AF over a total follow up of 14.3 patient years (AF recurrence rate of 2.2/patient year). The difference between these $\mathrm{AF}$ recurrence rates was significant $(\mathrm{p}<0.001)$.

In a multivariable Cox regression model that considered age, sex, duration of AF, coronary artery disease, hypertension, left ventricular hypertrophy, left atrial dimension, and CRP, the independent predictors of AF recurrence were CRP, female sex, and left atrial diameter. The adjusted hazard ratio comparing the upper 50th centile of CRP with the lower 50th centile of CRP was 2.0 (95\% confidence interval (CI) 1.2 to

Table 1 Baseline characteristics of study patients according to recurrence of atrial fibrillation (AF) after cardioversion

\begin{tabular}{llll}
\hline Characteristic & $\begin{array}{l}\text { No AF recurrence } \\
\text { (n=36) }\end{array}$ & $\begin{array}{l}\text { AF recurrence } \\
\text { (n=75) }\end{array}$ & p Value \\
\hline Age (years) & $66.5(12.8)$ & $67.7(10.7)$ & 0.6 \\
Women (n) & $17 \%(6)$ & $32 \%(24)$ & 0.09 \\
Duration of AF (days) & $203(345)$ & $222(479)$ & 0.9 \\
CAD (n) & $39 \%(14)$ & $40 \%(30)$ & 0.9 \\
Hypertension (n) & $71 \%(26)$ & $56 \%(42)$ & 0.05 \\
LVEF (\%) & $0.50(0.13)$ & $0.46(0.13)$ & 0.2 \\
LVH (n) & $56 \%(20)$ & $36 \%(27)$ & 0.06 \\
LA diameter (cm) & $4.3(0.9)$ & $4.7(0.9)$ & 0.06 \\
Antiarrhythmic drug & & $36(48 \%)$ & 0.5 \\
$\quad$ Amiodarone & $15(42 \%)$ & $17(23 \%)$ & \\
Flecainide & $12(33 \%)$ & $11(15 \%)$ & \\
Sotalol & $2(6 \%)$ & $5(7 \%)$ & \\
Propafenone & $5(14 \%)$ & $3(4 \%)$ & \\
Dofetilide & $2(6 \%)$ & $1(1.3 \%)$ & \\
Procainamide & 0 & $1(1.3 \%)$ & \\
$\quad$ Quinidine & 0 & $1(1.3 \%)$ & \\
Disopyramide & 0 & $6.34(12)$ & 0.002 \\
CRP (mg/l) & & $3.95(2.19-6.13)$ & 0.002 \\
$\quad$ Mean & $3.25(3.7)$ & $1.81(0.78-4.17)$ & \\
Median & &
\end{tabular}

Data are mean (SD), number (\%), or median (interquartile range). $C A D$, coronary artery disease; CRP, $C$ reactive protein; LA, left atrium; LVEF, left ventricular ejection fraction; LVH, left ventricular hypertrophy.
Table 2 Characteristics of study patients according to lower and upper 50th centiles of CRP

\begin{tabular}{|c|c|c|c|}
\hline Characteristic & $\begin{array}{l}\text { CRP } \leqslant 3.09 \mathrm{mg} / \mathrm{l} \\
(\mathrm{n}=56)\end{array}$ & $\begin{array}{l}\text { CRP }>3.09 \mathrm{mg} / \mathrm{l} \\
(\mathrm{n}=55)\end{array}$ & p Value \\
\hline Age (years) & 66.8 & 67.9 & 0.6 \\
\hline Women (n) & $27 \%(15)$ & $27 \%(15)$ & 0.9 \\
\hline Duration of $\mathrm{AF}$ (days) & 157 (294) & $276(546)$ & 0.3 \\
\hline CAD (n) & $30 \%(17)$ & $49 \%(27)$ & 0.05 \\
\hline Hypertension (n) & $67 \%(38)$ & $57 \%(31)$ & 0.3 \\
\hline LVEF (\%) & $0.49(0.13)$ & $0.46(0.13)$ & 0.3 \\
\hline $\mathrm{LVH}(\mathrm{n})$ & $43 \%(24)$ & $42 \%(23)$ & 0.9 \\
\hline LA diameter $(\mathrm{cm})$ & $4.5(0.9)$ & $4.7(0.9)$ & 0.3 \\
\hline $\mathrm{AF}$ recurrence rate & $2.2 /$ patient year & 4.9/patient year & $<0.001$ \\
\hline
\end{tabular}

3.2, $\mathrm{p}=0.007$ ), whereas the corresponding estimates for female sex and left atrial diameter (per centimetre increase) were 2.1 (95\% CI 1.2 to $3.5, \mathrm{p}=0.006)$ and 1.4 (95\% CI 1.1 to $1.8, \mathrm{p}=0.02)$, respectively. No other variables were retained in the final model. Figure 1 presents adjusted survival curves according to CRP concentration.

\section{DISCUSSION}

Previous studies have shown that younger age, smaller left atrial size, and shorter duration of AF are predictors of sinus rhythm maintenance. ${ }^{4}$ However, no biochemical marker has been shown to correlate with AF recurrence and many of the recurrences are thought to be secondary to electrical remodelling. ${ }^{910}$ The results of this study indicate that increased CRP concentrations are associated with higher rates of AF recurrence after cardioversion in patients with persistent AF taking antiarrhythmic drugs.

Atrial remodelling may result from several factors. These include chronic haemodynamic factors, such as hypertension and valvar heart disease, electrophysiological factors, and inflammation. Inflammation has been shown to play a part in postoperative AF. Bruins et al ${ }^{11}$ reported that the time of peak incidence of postoperative atrial arrhythmias correlates with a peak in CRP and CRP-complement complexes. In nonpostoperative AF, histological studies have shown inflammatory changes and increased fibrosis in the atrial biopsies of patients with lone AF. ${ }^{6}$ In addition, Chung et al found that CRP concentrations are higher in patients with AF than in

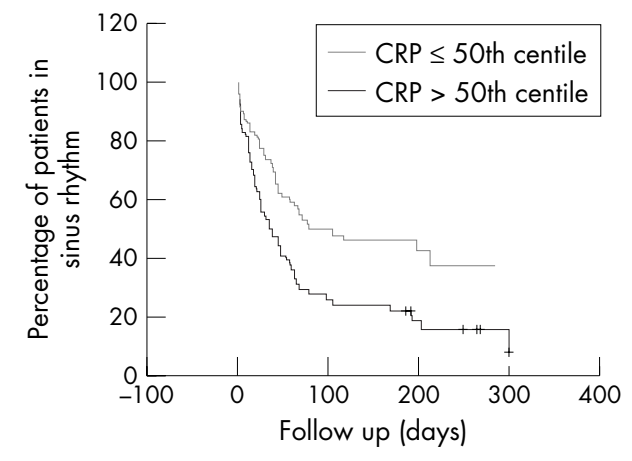

\begin{tabular}{lccccccc}
\hline Follow up (days) & & 0 & 50 & 100 & 150 & 200 & 250 \\
\hline \multirow{2}{*}{ CRP $\leq 50$ th centile } & Events & 0 & 23 & 5 & 6 & 1 & 1 \\
& At risk & 56 & 31 & 20 & 16 & 8 & 4 \\
\hline \multirow{2}{*}{ CRP > 50th centile } & Events & 0 & 34 & 6 & 1 & 2 & 0 \\
& At risk & 55 & 17 & 10 & 7 & 4 & 3 \\
\hline
\end{tabular}

Figure 1 Adjusted survival curves (percentage of patients in sinus rhythm over time) according to $C$ reactive protein (CRP) concentration. 
healthy controls. These authors also found that CRP concentrations were higher in patients with persistent AF than in those who have paroxysmal AF, suggesting that inflammation may promote the persistence of AF. ${ }^{7}$

Recently studies have shown that the use of antiinflammatory agents is associated with a decreased incidence of AF. In a recent study by Cheruku et al, ${ }^{12}$ the use of the nonsteroidal anti-inflammatory drug ketolorac significantly reduced AF after coronary artery bypass grafting. This was attributed to a decrease in inflammation caused by the nonsteroidal anti-inflammatory drug. ${ }^{12}$ Statins, which have been shown to decrease inflammation, ${ }^{13}$ also decreased the incidence of $\mathrm{AF}$ in recent studies in both animal models and humans. ${ }^{14-17}$ In addition, recent studies have shown that angiotensin converting enzyme inhibitors may decrease the incidence of $\mathrm{AF}$ in patients with reduced left ventricular ejection fraction. ${ }^{18}$ Furthermore, angiotensin converting enzyme inhibitors have recently been shown in to decrease inflammation. ${ }^{19-22}$ Madrid et $a l^{23}$ reported that adding the angiotensin receptor blocker irbesartan to amiodarone enhanced the probability of remaining in non-sinus rhythm after cardioversion of patients with persistent AF. This effect was attributed to the possible beneficial effects of blockade of the angiotensin receptor on apoptosis, fibrosis, and electrical remodelling. ${ }^{24-26}$ These results suggest that inflammation may have a causal role in the pathogenesis of AF.

\section{Limitations}

This was an observational study of consecutive patients taking antiarrhythmic drugs who underwent successful cardioversion. The period over which data were collected was arbitrarily selected. As there were no good prior data, sample size could not be estimated. However, CRP is a marker of inflammation and there is evidence to suggest that inflammation may have a causal role in some forms of AF. However, because this was an observational study, determining causation was problematic.

In our dataset, duration of $\mathrm{AF}$ was not strongly related to AF recurrence or to CRP concentration. However, it is important to note that duration of $\mathrm{AF}$ in this study refers to how long the patient had been in AF at the time of the cardioversion and not to the duration since AF was first detected. We did not have reliable data on when AF was first diagnosed and so could not take this into account with our analysis.

Lastly, as with all observational studies, confounding caused by factors that were not accounted for is possible. We did restrict our study sample to patients taking antiarrhythmic drugs; although this was not a bias, it may affect the generalisability of the study results.

\section{Conclusion}

This study shows that CRP concentrations independently predict recurrence of persistent AF after cardioversion. As was the case in previous studies, from our results we cannot conclude that CRP has a causal role or that AF has an inflammatory basis. ${ }^{7}$ Nevertheless, among this patient population we saw significantly higher CRP concentrations in the recurrence group than in the non-recurrence group. Inflammation may be more pathogenetic in promoting persistence of AF as Chung et $\mathrm{al}^{7}$ pointed out.

Increased CRP concentration may be a marker of more extensive atrial remodelling that may lead to lower effectiveness of antiarrhythmic drugs. Whether treatments that reduce inflammation and CRP are useful in treating patients with AF warrants further study.

\section{Authors' affiliations}

O Wazni, D O Martin, N F Marrouche, M Shaaraoui, M K Chung,

S Almahameed, R A Schweikert, W I Saliba, A Natale, The Center for Atrial Fibrillation, Cleveland Clinic Foundation, Cleveland, Ohio, USA

\section{REFERENCES}

1 Wyse DG, Waldo AL, DiMarco JP, et al. A comparison of rate control and rhythm control in patients with atrial fibrillation. N Engl J Med 2002;347:1825-33

2 Waldo AL. Management of atrial fibrillation: the need for AFFIRMative action. AFFIRM investigators. Atrial fibrillation follow-up investigation of rhythm management. Am J Cardiol 1999;84:698-700.

3 Van Gelder IC, Tuinenburg AE, Schoonderwoerd BS, et al. Pharmacologic versus direct-current electrical cardioversion of atrial flutter and fibrillation. Am J Cardiol 1999:84:147R-51R.

4 Van Gelder IC, Crijns HJ, Van Gilst WH, et al. Prediction of uneventful cardioversion and maintenance of sinus rhythm from direct-current electrical cardioversion of chronic atrial fibrillation and flutter. Am J Cardiol $1991 ; 68: 41-6$

5 Falk RH. Atrial fibrillation. N Engl J Med 2001;344:1067-78.

6 Frustaci A, Chimenti C, Bellocci F, et al. Histological substrate of atrial biopsies in patients with lone atrial fibrillation. Circulation 1997;96:1180-4.

7 Chung MK, Martin DO, Sprecher D, et al. C-reactive protein elevation in patients with atrial arrhythmias: inflammatory mechanisms and persistence of atrial fibrillation. Circulation 2001;104:2886-91.

8 Maldonado G, Greenland S. Simulation study of confounder-selection strategies. Am J Epidemiol 1993;138:923-36.

9 Allessie MA, Boyden PA, Camm AJ, et al. Pathophysiology and prevention of atrial fibrillation. Circulation 2001;103:769-77.

10 Wiiffels MC, Kirchhof CJ, Dorland R, et al. Atrial fibrillation begets atrial fibrillation: a study in awake chronically instrumented goats. Circulation 1995;92:1954-68

11 Bruins $\mathbf{P}$, te Velthuis $\mathrm{H}$, Yazdanbakhsh AP, et al. Activation of the complement system during and after cardiopulmonary bypass surgery: postsurgery activation involves $\mathrm{C}$-reactive protein and is associated with postoperative arrhythmia. Circulation 1997;96:3542-8.

12 Cheruku KK, Ghani A, Ahmad F, et al. Efficacy of nonsteroidal antiinflammatory medications for prevention of atrial fibrillation following coronary artery bypass graft surgery. Prev Cardiol 2004;7:13-8.

13 Shishehbor MH, Brennan ML, Aviles RJ, et al. Statins promote potent systemic antioxidant effects through specific inflammatory pathways. Circulation 2003;108:426-31.

14 Korantzopoulos P, Kountouris E, Kolettis T, et al. Anti-inflammatory and antioxidant actions of statins may favorably affect atrial remodeling in atrial fibrillation. Am J Cardiol 2004;93:1200.

15 Korantzopoulos $\mathbf{P}$, Kokkoris S. The antioxidant effects of statins may extend beyond atherosclerosis: potential benefits for atrial fibrillation and heart failure. Atherosclerosis 2004;175:187.

16 Siu CW, Lau CP, Tse HF. Prevention of atrial fibrillation recurrence by statin therapy in patients with lone atrial fibrillation after successful cardioversion. Am J Cardiol 2003;92:1343-5.

17 Young-Xu Y, Jabbour S, Goldberg R, et al. Usefulness of statin drugs in protecting against atrial fibrillation in patients with coronary artery disease. Am J Cardiol 2003;92: 1379-83.

18 Pedersen OD, Bagger H, Kober L, et al. Trandolapril reduces the incidence of atrial fibrillation after acute myocardial infarction in patients with left ventricular dysfunction. Circulation 1999;100:376-80.

19 Brull DJ, Sanders J, Rumley A, et al. Impact of angiotensin converting enzyme inhibition on post-coronary artery bypass interleukin 6 release. Heart 2002;87:252-5.

20 Hernandez-Presa $M$, Bustos $C$, Ortego $M$, et al. Angiotensin-converting enzyme inhibition prevents arterial nuclear factor-kappa $B$ activation, monocyte chemoattractant protein-1 expression, and macrophage infiltration in a rabbit model of early accelerated atherosclerosis. Circulation 1997; 95: 1532-41.

21 Hernandez-Presa MA, Bustos C, Ortego M, et al. ACE inhibitor quinapril reduces the arterial expression of NF-kappaB-dependent proinflammatory factors but not of collagen I in a rabbit model of atherosclerosis. Am J Pathol 1998; 153:1825-37

22 Kranzhofer R, Schmidt J, Pfeiffer CA, et al. Angiotensin induces inflammatory activation of human vascular smooth muscle cells. Arterioscler Thromb Vasc Biol 1999; 19:1623-9.

23 Madrid AH, Bueno MG, Rebollo JM, et al. Use of irbesartan to maintain sinus rhythm in patients with long-lasting persistent atrial fibrillation: a prospective and randomized study. Circulation 2002;106:331-6.

24 Fortuno MA, Ravassa S, Etayo JC, et al. Overexpression of Bax protein and enhanced apoptosis in the left ventricle of spontaneously hypertensive rats: effects of AT1 blockade with losartan. Hypertension 1998;32:280-6.

25 Lopez B, Querejeta R, Varo N, et al. Usefulness of serum carboxy-terminal propeptide of procollagen type I in assessment of the cardioreparative ability of antihypertensive treatment in hypertensive patients. Circulation 2001;104:286-91.

26 Nakashima H, Kumagai K, Urata H, et al. Angiotensin II antagonist prevents electrical remodeling in atrial fibrillation. Circulation 2000;101:2612-7. 